\title{
Bionanoconjugates of tyrosinase and peptide-derivatised gold nanoparticles for biosensing of phenolic compounds
}

\author{
J. Cortez $\cdot$ E. Vorobieva $\cdot$ D. Gralheira $\cdot$ \\ I. Osório $\cdot$ L. Soares $\cdot$ N. Vale $\cdot$ E. Pereira $\cdot$ \\ P. Gomes $\cdot$ R. Franco
}

Received: 24 May 2010/ Accepted: 16 September 2010/Published online: 6 October 2010

(C) Springer Science+Business Media B.V. 2010

\begin{abstract}
Bionanoconjugates of the enzyme tyrosinase (TYR) and gold nanoparticles (AuNPs) functionalised with a peptide (CALNN) were produced in solution and characterised. The formation of stable TYR-AuNP:CALNN bionanoconjugates (BNCs) was supported by a decrease of the surface charge of the BNCs as determined by $\zeta$-potential and an increase in hydrodynamic diameter as determined by Dynamic Light Scattering (DLS). UV/Vis studies of $\mathrm{pH}$-induced aggregation revealed distinct protonation patterns for the BNCs when compared with AuNP:CALNN alone, further substantiating BNC formation. Activity studies of the BNCs for the reduction of di-phenols in solution indicated that TYR not only remains active after conjugation, but interestingly its activity in the BNCs is higher than for the free enzyme. In conclusion,
\end{abstract}

J. Cortez $(\bowtie) \cdot$ E. Vorobieva · D. Gralheira ·

I. Osório - R. Franco

REQUIMTE, Departamento de Química, Faculdade de

Ciências e Tecnologia da Universidade Nova de Lisboa,

Campus de Caparica, 2829-516 Caparica, Portugal

e-mail: j.cortez@dq.fct.unl.pt

L. Soares · E. Pereira

REQUIMTE, Departamento de Química e Bioquímica, Faculdade de Ciências da Universidade do Porto,

R. Campo Alegre, 687, 4169-007 Porto, Portugal

N. Vale $\cdot$ P. Gomes

CIQUP, Departamento de Química e Bioquímica, Faculdade de Ciências da Universidade do Porto, R. Campo Alegre, 687, 4169-007 Porto, Portugal
AuNP:CALNN can provide a suitable platform for the immobilisation of TYR, leading to BNCs with increased enzyme activity and a wider $\mathrm{pH}$ working range, with promising uses in electrochemical biosensors for the detection of mono- and di-phenolic compounds.

Keywords Tyrosinase - Gold nanoparticle · Bionanoconjugate $\cdot$ Biosensor $\cdot$ Environmental pollutants

\section{Introduction}

Environmental sensors are gaining importance because of ever-growing environmental concerns and their ability to provide rapid information on the identification and/or quantification of contaminants or toxic compounds in samples. In particular, there is a great need for systems of analysis and detection of environmental pollutants such as pesticides (Vamvakaki and Chaniotakis 2007; Viveros et al. 2006).

Enzyme electrodes present themselves as very promising tools for screening purposes or alarm signalling (Dzyadevych et al. 2005; Rogers 1995). Amongst enzyme biosensors, those based on the enzyme tyrosinase for the detection of phenolic compounds have been attracting great interest for fast and simple detection of pesticides (Kim et al. 2008a, b, c; Vidal et al. 2006) or phenolic compounds in food (Huang et al. 1992). 
Tyrosinases (TYRs) are copper-containing metalloproteins that can be found in fungi, yeast, plants and mammals (Jolivet et al. 1998). TYRs are bifunctional enzymes, catalysing two distinct reactions: (i) the $o$-hydroxylation of monophenols (cresolase or monophenolase activity, EC 1.14.18.1) and (ii) the oxidoreduction of ortho-diphenols to ortho-quinones (catecholase or diphenolase activity, EC 1.10.3.1), utilising molecular oxygen as an electron acceptor that is reduced to water (Robb 1984; Selinheimo et al. 2009; Sfinchez-Ferrer et al. 1995; VanGelder et al. 1997). Monophenolase activity is known to be the initial rate-determining reaction (Robb 1984; Rodriguez-Lopez et al. 1992). TYR can thus accept both mono- and diphenols as substrates, offering great potential for the development of biosensors (Abdullah et al. 2006; Hedenmo et al. 1997; Kim et al. 2008b, c) for the detection of phenolic compounds (from pollutants, pesticides) for e.g. in wastewaters.

Recent studies demonstrated the improvement on the performance of enzyme electrodes with the introduction of gold nanoparticles (AuNPs) (Kim et al. 2008c; Salimi et al. 2007; Willner et al. 2007). The use of AuNPs is a very promising area in the development of biosensors because of their unique properties such as high surface area, size-dependent physical properties, and ease of synthesis and functionalisation (Baptista et al. 2008). Owing to these properties, AuNPs can be used as tuneable scaffolds for the immobilisation of sensing proteins, and thus for the fabrication of biosensors with redox enzymes such as TYR.

The conjugation of enzymes with AuNPs can lead to the retention or even to an increase of their biological stability/activity (You et al. 2006). Electron transfer between the catalytic sites of immobilised enzymes and the electrode materials is facilitated, improving the analytical sensitivity and selectivity of the biosensors and often obviating the need for enzyme mediators (Kim et al. 2008c; Willner et al. 2007; Yu et al. 2003). These properties have led to an intensive study of AuNPs in the construction of electrochemical biosensors with enhanced analytical performance with respect to other biosensor designs (Baron et al. 2007; Pingarron et al. 2008).

Kim et al. (2008a, b, c) reported a TYR phenol sensor assembled by electrochemical deposition of AuNPs on an electrode surface, modified by a protective selfassembled monolayer (SAM) of cysteamine and followed by the immobilisation of TYR. The deposition of
AuNPs was found to improve the current response of the TYR-based electrode, which was capable of detecting pesticides such as atrazine and ziram in the concentration range of $0.001-0.5 \mathrm{ng} \mathrm{mL}^{-1}$.

Strategies for gold surface modification include the formation of monolayers of alkanethiols, to provide a convenient, flexible and simple interface system constituting a 'biologically friendly' environment for the adsorption of proteins, and minimising any unfavourable interactions between the protein and the gold surface (Love et al. 2005). Nonetheless, the use of AuNPs as a scaffold for enzyme immobilisation is still often impaired by loss of bioactivity.

Peptides show great potential in the control of surface charge and surface chemistry of AuNPs (Levy 2006), allowing the optimisation of the interaction with the selected protein by tailoring the amino acids sequence used. Gold nanoparticles capped with a peptide monolayer have been shown to acquire properties similar to proteins (Levy 2006), presenting a favourable environment for the attachment of proteins and the preservation of their bioactivity.

Our approach was based on the preparation of AuNPs with an average diameter of $c a .11 \mathrm{~nm}$, derivatised with the pentapeptide Cys-Ala-Leu-Asn-Asn (CALNN) to probe the effect of AuNP surface chemistry on TYR activity and to produce a suitable platform for the assembly of active TYR-AuNP:CALNN conjugates. The CALNN peptide was designed so as to exhibit a thiol group from an $\mathrm{N}$-terminal cysteine for binding onto gold nanoparticles, and include a hydrophobic region made of alanine and leucine residues to promote self-assembly at the nanoparticle surface (Levy et al. 2004). The $C$-terminus comprises two uncharged and hydrophilic asparagine residues for interaction with TYR and to ensure nanoparticle stability and solubility in water. This peptide forms a compact monolayer on the surface of the nanoparticles (Levy 2006; Levy et al. 2004).

By functionalising AuNPs with CALNN (producing AuNP:CALNN), we provide TYR with a platform that mimics the chemistry of a protein surface for TYR binding, allowing interactions with the nanoparticles that resemble naturally occurring protein-protein interactions systems. The predominant interactions between the CALNN capping and the outer amino acid residues of TYR are expected to be electrostatic interactions and hydrogen-bonds. These types of weak interactions are preferred to covalent 
binding, which is known to be potentially damaging to the protein-active folding. At $\mathrm{pH} 7.0$, the peptidecapped AuNPs present a global negative charge, as confirmed by zeta potential analysis, and will interact with positively charged residues of TYR. The side chains of the asparagine residues are hydrophilic and uncharged, but will facilitate the retention of the protein conformation when immobilised by weak interactions with the protein, but also by maintaining high water solubility. The formation of a compact and stable peptide layer at the surface of the AuNP is expected to prevent direct interactions between cysteine residues of TYR and the gold surface, avoiding perturbations to the protein conformation that such strong interactions would cause.

Our aims were the retention of enzyme bioactivity in the bionanoconjugates (BNCs) and the improvement of stability and sensitivity of the biosensing probe in posterior sensor assembly.

The BNCs formed in aqueous solution were characterised by Dynamic Light Scattering (DLS) and $\zeta$-potential measurements, and were tested for their enzymatic activity in relation to the free enzyme.

We have demonstrated the formation of individual BNCs of TYR and AuNP:CALNN, with the nanoparticle surface becoming saturated with enzyme for a ratio of 50 protein molecules per nanoparticle. We also found that the BNCs retained TYR activity and, when compared to the free enzyme, presented similar or higher enzymatic activity, in the $\mathrm{pH}$ range from 4.0 to 8.0.

\section{Materials and methods}

Materials

Tyrosinase from Agaricus bisporus was obtained from Sigma-Aldrich and dissolved in $10 \mathrm{mM}$ phosphate buffer, $\mathrm{pH}$ 7.0. The enzyme was used as obtained, with no purification steps to retain maximum activity. Possible small molecules used for stabilization were removed by ultrafiltration, using Amicon Ultra $100 \mathrm{kDa}$ cut-off membrane filters from Milipore. All chemicals used were purchased from Sigma-Aldrich and were of analytical grade. Aqueous solutions, including phosphate buffer, were prepared with MilliQ water $\left(18 \mathrm{M} \Omega \mathrm{cm}^{-1}\right)$.
The pentapeptide CALNN was synthesised with a cysteine amino acid at the $N$-terminus for binding onto the gold nanoparticles. $N^{\alpha}$-Fmoc-protected amino acids and Fmoc-Asn(Trt)-Wang resin used for peptide synthesis were from Novabiochem (VWR International), whereas solvents ( $N, N$-dimethylformamide, DMF; and dichloromethane, DCM) as well as reagents for amino acid coupling ( $N, N^{\prime}$-diispropylcarbodiimide, DIC) and deprotection (piperidine) were all from Sigma-Aldrich.

Methods

\section{Tyrosinase activity assay}

The activity of TYR was determined as described by Robb (1984) with few modifications, in the presence of $1.0 \mathrm{mM}$ of the substrate 4-methyl-catechol (MC). The assays were carried out in $10 \mathrm{mM}$ phosphate buffer $\mathrm{pH} 7.0$ at $25{ }^{\circ} \mathrm{C}$ for an incubation time of 5 min and for known amounts of enzyme or BNC. The activity of the enzyme was determined by monitoring the formation of the reaction product (o-4-methyl-benzoquinone) at $400 \mathrm{~nm}$ by UV/Vis spectrophotometry (UNICAM, model UV2, UV/ Visible spectrophotometer, using quartz cells from Hellma, Germany).

The activity was measured $24 \mathrm{~h}$ after preparation of the BNCs (stored at $4{ }^{\circ} \mathrm{C}$ ) and for comparison an enzyme solution at the same concentration was also left at $4{ }^{\circ} \mathrm{C}$ for $24 \mathrm{~h}$ before determining its activity. The same procedure was followed in the determination of TYR activity of the BNC solutions at different pHs. The enzyme activity was normalised to the free enzyme.

\section{Pentapeptide synthesis}

The peptide ( $C$-terminal carboxyl) was assembled by classical Fmoc/tBu solid phase peptide synthesis methodologies (Fields and Noble 1990), on a preloaded Fmoc-Asn(Trt)-Wang resin (Wang 1973). In brief, after removal of the Fmoc-protecting group from the pre-loaded resin with $20 \%$ piperidine in DMF, the remaining four amino acids were sequentially coupled in the $C \rightarrow N$ direction by means of standard coupling + deprotection cycles. All the coupling reactions were carried out during $c a$. 60 min, using 5-molar excesses of both the relevant 
Fmoc-protected amino acid and the coupling reagent (DIC), and using DCM or DCM/DMF mixtures as solvents. Deprotection reactions were done by treatment of the resin with $20 \%$ piperidine in DMF $(1 \mathrm{~min}+20 \mathrm{~min})$. Both coupling and deprotection were repeated until completion, which was verified by the ninhydrin or Kaiser assay (Kaiser et al. 1970). After being assembled, the peptides were fully deprotected and cleaved from the resin by acidolysis $(3 \mathrm{~h})$ of the peptidyl-resin using a trifluoroacetic acid (TFA)-based cocktail (Fields and Noble 1990) containing water and triisopropylsilane (TIS) as scavengers (TFA/ $\mathrm{H}_{2} \mathrm{O} / \mathrm{TIS}$ 95:2.5:2.5 v/v/v). The crude product was purified by reverse-phase liquid chromatography, and the final product was analysed by HPLC, ESI-IT MS and amino acid analysis (AAA).

\section{Gold nanoparticle synthesis and preparation} of TYR-AuNP: CALNN bionanoconjugates

Colloidal AuNPs were synthesised by the traditional citrate method by Turkevich, with minor alterations (Baptista et al. 2005; Kimling et al. 2006). The as-prepared AuNPs were then functionalised with the peptide CALNN (AuNP:CALNN) by overnight incubation at a molar ratio of 1:120 at room temperature. The success of the capping exchange reaction was evaluated by X-ray photoelectron spectroscopy (XPS). Samples were prepared by removing the excess of citrate and CALNN by five steps of centrifugation/ resuspension in water. The pellet obtained in the last centrifugation step was deposited in a carbon disk, and analysed in a VG Scientific Escalab 200A with nonmonochromatic $\mathrm{Al} \mathrm{K}$ radiation $(1486.6 \mathrm{eV}$ ) $)$ at the Centro de Materiais da Universidade do Porto (CEMUP).

The TYR-AuNP:CALNN BNCs were prepared by incubating overnight at $4{ }^{\circ} \mathrm{C}$ in $10 \mathrm{mM}$ phosphate buffer $\mathrm{pH} 7.0$ the as-prepared AuNP:CALNN diluted to $1 \mathrm{nM}$ (estimated using $\varepsilon_{520 \mathrm{~nm}}=2.3 \times 10^{8} \mathrm{M}^{-1} \mathrm{~cm}^{-1}$ ) (Baptista et al. 2005) with MilliQ water with appropriate amounts of TYR to obtain BNCs with the desired [TYR]/[AuNP:CALNN] molar ratio. TYR concentration (in $\mathrm{mg} \mathrm{mL}^{-1}$ ) was determined by the bicinchoninic acid assay with bovine serum albumin as standard (Smith et al. 1985). Conversion of TYR concentration values from $\mathrm{mg} \mathrm{mL}^{-1}$ to molar was based on the native form of the enzyme. It should, however, be noted that the active form of the enzyme is the dissociated form, concentration of which is difficult to determine with certainty.

The BNCs were washed by ultrafiltration using a membrane with a cut off of $100 \mathrm{kDa}$ at 5,000 rpm for $5 \mathrm{~min}$ at $4{ }^{\circ} \mathrm{C}$, to guarantee the removal of any unbound protein and any possible interfering contaminants. The BNCs were stored at $4{ }^{\circ} \mathrm{C}$ until use.

\section{Effect of $\mathrm{pH}$ on the bionanoconjugates}

Bionanoconjugate $\mathrm{pH}$-dependence studies were performed at $25{ }^{\circ} \mathrm{C}$ by UV/vis spectroscopy. The $\mathrm{pH}$ of $1 \mathrm{~mL}$ of BNC solution (1 $\mathrm{nM}$ AuNP:CALNN and 85 $\mathrm{U} \mathrm{mL}^{-1}$ of TYR) was adjusted with solutions of $0.01 \mathrm{M} \mathrm{HCl}$ and $0.01 \mathrm{M} \mathrm{NaOH}$ to study the effect of $\mathrm{pH}$ on the stability of the BNCs. UV/Vis spectra of each solution were measured immediately after $\mathrm{pH}$ adjustment. The absorption values were normalised taking into account the volume of solution added to adjust the $\mathrm{pH}$ of the solution.

To determine the effect of $\mathrm{pH}$ on the TYR activity of the BNCs, for each tube after $\mathrm{pH}$ adjustment, enzyme substrate (MC) was added to a final concentration of $1.0 \mathrm{mM}$, allowed to incubate for $5 \mathrm{~min}$ and the UV/Vis spectra of each solution measured.

\section{Stability of the bionanoconjugates}

Bionanoconjugates of 1nM AuNP:CALNN and 85 $\mathrm{U} \mathrm{mL}^{-1}$ TYR were prepared and their activity determined (for 4-methyl catechol, as described above) on the next day after preparation and every week subsequently up to 6 weeks. The BNCs were kept at $4{ }^{\circ} \mathrm{C}$ until analysis. This was compared with the stability of the free enzyme and under the same conditions. The results are expressed as the percentage change from the original activity, for either the $\mathrm{BNC}$ or the free enzyme.

\section{Light scattering analysis}

Measurements of DLS and zeta potential of the nanoparticles obtained with different concentrations of TYR were used as a tool to demonstrate the formation of TYR-AuNP:CALNN conjugates. With this purpose, solutions of BNCs were prepared for 1 $\mathrm{nM}$ of AuNP and ratios of 1:5, 1:10, 1:25, 1:50, 1:100 e 1:200 of TYR concentration, for a final volume of $1 \mathrm{~mL}$ in $10 \mathrm{mM}$ phosphate buffer $\mathrm{pH}$ 7, using MilliQ 
water as necessary. These solutions were stored at $4{ }^{\circ} \mathrm{C}$ and analysed the next day.

Dynamic light scattering and $\zeta$-potential measurements were performed in a Zetasizer Nano-ZS from Malvern Instruments. A 4-mW He-Ne laser $(633 \mathrm{~nm})$ was used with a fixed $173^{\circ}$ (DLS) or $17^{\circ}$ (zetapotential) scattering angle. All measurements were carried out at $25{ }^{\circ} \mathrm{C}$, and the experiments were started only after the sample reached thermal equilibrium ( $5 \mathrm{~min}$ ). One millilitre of the samples prepared was transferred to a disposable sizing cuvette for DLS (Sarsted, Germany) or to a disposable zeta cell for $\zeta$-potential measurements (Malvern, UK).

In DLS, each sample was measured three times and each measurement consisted of 13-15 acquisitions. Using the Malvern software package for the DLS measurements, the autocorrelation function of the scattered intensity was analysed by means of the inverse Laplace transformation program CONTIN, and the hydrodynamic diameter, $\mathrm{d}(H)$, was calculated using the Stokes-Einstein equation:

$\mathrm{d}(H)=\frac{k T}{3 \pi \eta D}$

where $\mathrm{d}(H)$ is the hydrodynamic diameter, $D$ is the translational diffusion coefficient, $k$ is the Boltzman constant, $T$ is the absolute temperature, and $\eta$ is the viscosity.

In $\zeta$-potential, each sample was measured three times and each measurement consisted of 100 acquisitions. Repeated measurements allowed for error estimation. The $\zeta$-potential was determined using the Malvern software package through Henry's equation that relates electrophoretic mobility to $\zeta$-potential:

$U_{\mathrm{E}}=\frac{2 \varepsilon \zeta}{3 \eta} f(\kappa R)$

where $U_{\mathrm{E}}$ is the electrophoretic mobility, $\varepsilon$ and $\eta$ are the dielectric constant and viscosity of the solvent, respectively, and $\zeta$ is the $\zeta$-potential. Henry's function, $f(\kappa R)$, was approximated by the Smoluchowski approximation, $f(\kappa R)=1.5$, since the $\zeta$-potential measurements were performed in aqueous solution. Data obtained for different [TYR]/[AuNP:CALNN] ratios were fitted to a Langmuir adsorption isotherm (Eq. 3) using Excel 2007 Solver software.

The data were expressed as delta $\zeta$-potential, i.e. the $\zeta$-potential of the various BNCs relative to the potential of the AuNP:CALNN alone at $\mathrm{pH}$ 7.0.

\section{Results and discussion}

Characterisation of the CALNN-derivatised gold nanoparticles

The AuNP:CALNN nanoparticles presented a plasmon resonance band at $522 \mathrm{~nm}$, showing a 2-nm redshift in relation to the as-prepared citrate AuNPs. The determination of the hydrodynamic diameter by DLS presented a consistent value of $10.5 \pm 0.9 \mathrm{~nm}$, with a poly-dispersion index of $0.45 \pm 0.02$, while the citrate AuNPs had an average hydrodynamic diameter of $9.3 \pm 0.8 \mathrm{~nm}$ and a poly-dispersion index of $0.40 \pm 0.07$. Taken together, the changes observed by UV/Vis and DLS upon incubation of the NPs with the pentapeptide show that exchange of the capping agent was successful, and that the pentapeptide is more effective towards preventing the aggregation of AuNPs than citrate. In addition, XPS of the AuNP:CALNN nanoparticles after extensive washing show the presence of sulphur and nitrogen, thus confirming the presence of the pentapeptide as the capping agent.

The AuNP:CALNN colloidal solution was found to be stable towards aggregation between $\mathrm{pH} 4$ and 8 (Fig. 1). The UV-vis spectra presented almost no alterations from $\mathrm{pH} 8.0$ to 3.8 , but red-shifted abruptly to $630 \mathrm{~nm}$ for $\mathrm{pH} 3.5$ or lower. The plasmon band shift (noted by a reduction of the plasmonic band maximum at $522 \mathrm{~nm}$ and appearance of a band at $630 \mathrm{~nm}$ ) was visually detected by a change in colour of the solution from red to blue, corresponding to nanoparticle aggregation.

The inset in Fig. 1, is a graphical representation of the absorbance ratios between aggregated and nonaggregated species in solution. This representation allows the determination of a 'half-titration' point for the aggregation process, which was estimated as $\mathrm{pH}$ 3.4.

Characterisation of the tyrosinase bionanoconjugates

The TYR BNC exhibited a plasmon resonance band at $525 \mathrm{~nm}$, presenting a 3-nm red-shift relative to the AuNP:CALNN colloidal solution (Fig. 2).

The BNC solutions exhibited a distinct $\mathrm{pH}$-induced aggregation behaviour compared with AuNP:CALNN. The aggregation of the BNCs was indicated by a $20-\mathrm{nm}$ 
Fig. 1 UV/Vis spectra for the AuNP:CALNN as a function of $\mathrm{pH}$. Inset Variation in the ratios of absorbance for the plasmon resonance maxima in the aggregated and nonaggregated $\left(\mathrm{A}_{630 \mathrm{~nm}} / \mathrm{A}_{522 \mathrm{~nm}}\right)$ forms of AuNP:CALNN as a function of $\mathrm{pH}$

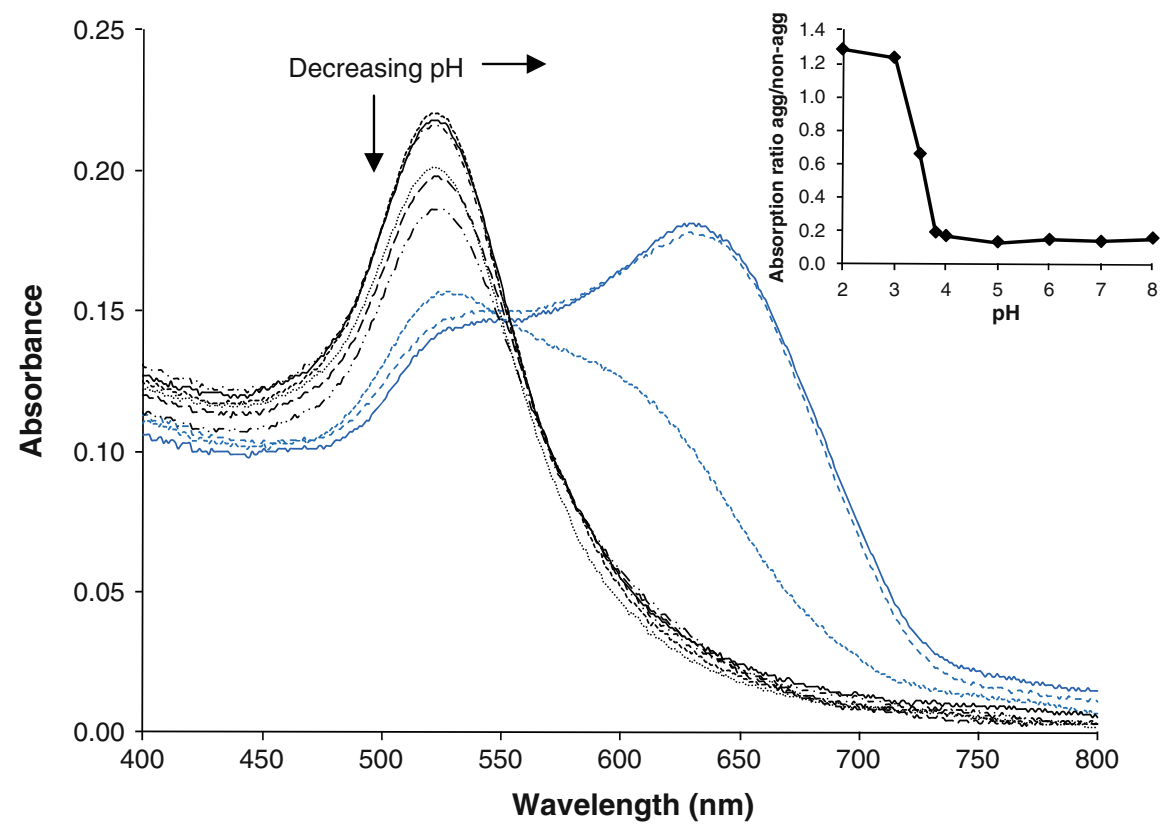

Fig. 2 UV/Vis spectra for the BNCs (prepared at a ratio of [TYR]/

[AuNP:CALNN] of 1:100) as a function of $\mathrm{pH}$. Inset Variation in the ratios of absorbance for the plasmon resonance maxima in the aggregated and nonaggregated $\left(\mathrm{A}_{545 \mathrm{~nm}} / \mathrm{A}_{525 \mathrm{~nm}}\right)$ forms of TYR-

AuNP:CALNN BNCs as a function of $\mathrm{pH}$



red-shift of the plasmonic band from 525 to $545 \mathrm{~nm}$, smaller than that observed for the pentapeptidefunctionalised AuNPs (Fig. 2). This difference in behaviour is probably related to the presence of a TYR protein corona enveloping the nanoparticle surface, and thus leading to a greater inter-particle distance in the aggregated form, compared with the AuNP:CALNN alone, and thus to a weaker coupling of the plasmon resonance between particles.
Aggregation of the BNCs occurred at a higher $\mathrm{pH}$ relative to AuNP:CALNN alone. That is at $\mathrm{pH} 5.0$ or 5.5 , a red-shift to $545 \mathrm{~nm}$ was observed. Interestingly, for $\mathrm{pH} 4.0$ or lower, the plasmon band blueshifted to $535 \mathrm{~nm}$. We propose that this shift might be caused by changes in the bound protein conformation or even unfolding (caused by low $\mathrm{pH}$ ) at the AuNPs surface, leading to an increase in distance between AuNPs when compared with aggregated forms 
between $\mathrm{pH} 5.0$ and 4.0, and thus to lower interactions between nanoparticles.

Figure 2 also shows a graphical representation of the absorbance ratios between aggregated and nonaggregated species in solution for the TYR conjugates. The 'half-titration' point for the aggregation process of the BNCs was estimated as $\mathrm{pH}$ 5.3, compared with pH 3.4 for AuNP:CALNN (Fig. 1). This difference demonstrates clearly distinct $\mathrm{pH}$-induced aggregation behaviour of the BNCs when compared with AuNP:CALNN alone, further supporting the presence of a TYR layer at the nanoparticle surface. This protein corona leads to a distinct surface charge and protonation behaviour compared with the nanoparticles alone and thus to aggregation at different $\mathrm{pH}$ levels.

Stability of the bionanoconjugates

The stability of the BNCs at $4{ }^{\circ} \mathrm{C}$ was tested every week for a total of 6 weeks and compared to that of the free enzyme (Fig. 3). The difference in stability was found to be marginally higher for the conjugates but was not statistically different to that of the free TYR (for $p<0.05$ ). The enzyme activity of the BNCs was stable for 6 weeks, with a loss of activity towards 4-methyl catechol of only $10 \%$. This indicates that these conjugates present a good level of storage stability for preparation and assembly of the biosensor at a later stage, suggesting that they will also be stable after immobilisation onto the electrode surface.
Light scattering analysis of the bionanoconjugates

\section{Zeta potential}

Measurements of $\zeta$-potential of the BNCs were obtained for different concentrations of TYR at the surface of AuNP:CALNN to further substantiate the formation of BNCs. This technique determines the electrical potential at the interfacial double layer of the conjugates and can be correlated to the amount of protein bound at the AuNP surface. Since surface potential is associated to the surface charge and the thickness of the double layer, the adsorption of protein will alter these parameters. As reported previously (Brewer et al. 2005; Gomes et al. 2008), it is expected that an increasing amount of protein bound to the nanoparticle surface will lead to a shift in the surface potential until a stable value is observed, indicating the nanoparticle surface is saturated with protein.

Bionanoconjugates with increasing concentrations of TYR (for a constant concentration of AuNPs) were analysed at $\mathrm{pH} 7.0$, at which the protein is stable and active, and no aggregation was observed (Fig. 2). The AuNPs:CALNN alone presented a $\zeta$-potential of $-47.7 \pm 0.3 \mathrm{mV}$, a value marginally higher than that obtained for citrate-stabilised AuNPs (Brewer et al. 2005; Gomes et al. 2008). The $\zeta$-potential of the free enzyme in the same conditions $(\mathrm{pH} 7.0$ and $25^{\circ} \mathrm{C}$ ) was $-17.5 \pm 0.5 \mathrm{mV}$.

In the studies of protein adsorption to gold nanoparticles at constant temperature, a Langmuir isotherm
Fig. 3 Storage stability of the bionanoconjugates (BNCs) compared with the free enzyme, expressed as percentage activity retained compared to the activity at day 1 of the respective preparation

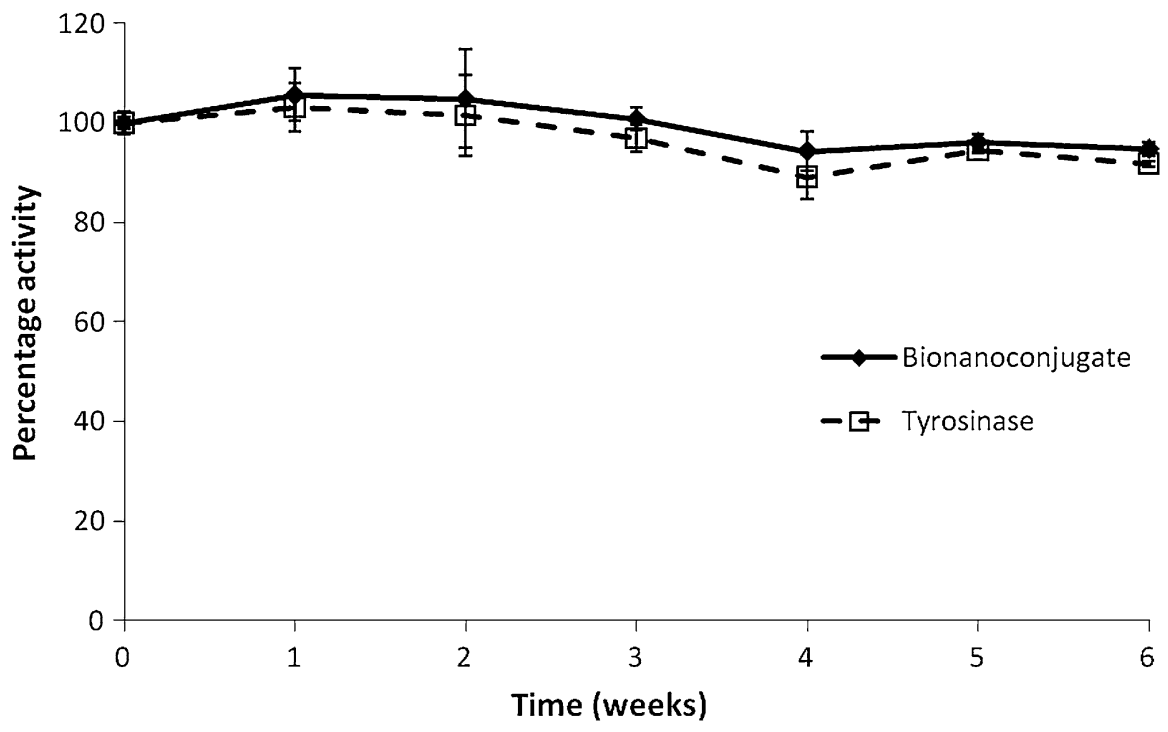


can be fitted to changes of $\zeta$-potential as a function of protein concentration at the AuNP surface (Brewer et al. 2005; Kaufman and 2007), as $\zeta$-potential can be related to the surface charge of the portion of the adsorbed protein molecule that is exposed to the solvent. As more protein is adsorbed at the AuNP surface, the formed BNC increases its surface potential away from the very negative value observed for the CALNN-stabilised AuNP, until a stable value is attained corresponding to homogeneous protein coverage, as observed in Fig. 4. This approach led to a Langmuir adsorption isotherm-type equation:

$\Delta \zeta=\frac{\Delta \zeta_{\max } K_{\mathrm{L}} R}{1+K_{\mathrm{L}} R}$

in which $\Delta \zeta$ is the variation in the $\zeta$-potential measured for a BNC with a surface coverage $R=[\mathrm{TYR}] /[\mathrm{AuNP}: \mathrm{CALNN}]$ in relation to the AuNP:CALNN alone; $\Delta \zeta_{\max }$ is the maximum value for $\Delta \zeta$-potential as $R$ increases; and $K_{\mathrm{L}}$ is a binding constant corresponding to the value of the inverse of the concentrations ratio, $1 / R$, for one-half of $\Delta \zeta_{\max }$.

Figure 4 illustrates the variation in $\zeta$-potential $(\Delta \zeta$ potential) of BNCs prepared with increasing ratios of [TYR]/[AuNP:CALNN] relative to the $\zeta$-potential of AuNP:CALNN alone. Increasing the ratios resulted in a shift from the $\zeta$-potential value of AuNP:CALNN alone, to a maximal variation $\left(\Delta \zeta_{\max }=23.1 \pm 0.2 \mathrm{mV}\right.$, as obtained from fitting to Eq. 3). Visual inspection of the graphical representation on Fig. 4 indicates that a maximal variation of the $\zeta$-potential is attained for a [TYR]/[AuNP:CALNN] ratio of approximately 50 ; and that no appreciable variation of the $\zeta$-potential was observed for higher concentration of protein. This plateau of the $\zeta$-potential values suggest that the AuNP surface becomes fully enveloped with a TYR monolayer, and that even for higher ratios of [TYR]/[AuNP:CALNN] no further protein adsorption occurs.

The binding constant obtained from the fitting of Eq. 3 to the experimental data was $K_{\mathrm{L}}=0.050$ \pm 0.005 , a value that is of the same order of magnitude as obtained for the formation of cytochrome c-AuNP BNCs (Gomes et al. 2008).

\section{Dynamic Light Scattering}

In order to corroborate the formation of the BNCs, measurements of the hydrodynamic diameter of the BNCs were obtained by DLS for different [TYR]/ [AuNP:CALNN] ratios. DLS determines the hydrodynamic diameter of the conjugates and can be correlated to the amount of protein bound at the AuNP surface, since this diameter increases upon binding of increasing amounts of protein. An increasing amount of protein bound to the nanoparticle surface is expected to lead to an increase in particle diameter until a stable value is observed, indicating that the nanoparticle
Fig. 4 Variation of the $\zeta$ potential of the bionanoconjugates (BNCs) in relation to AuNP:CALNN alone, as a function of the [TYR]/ [AuNP:CALNN] ratio

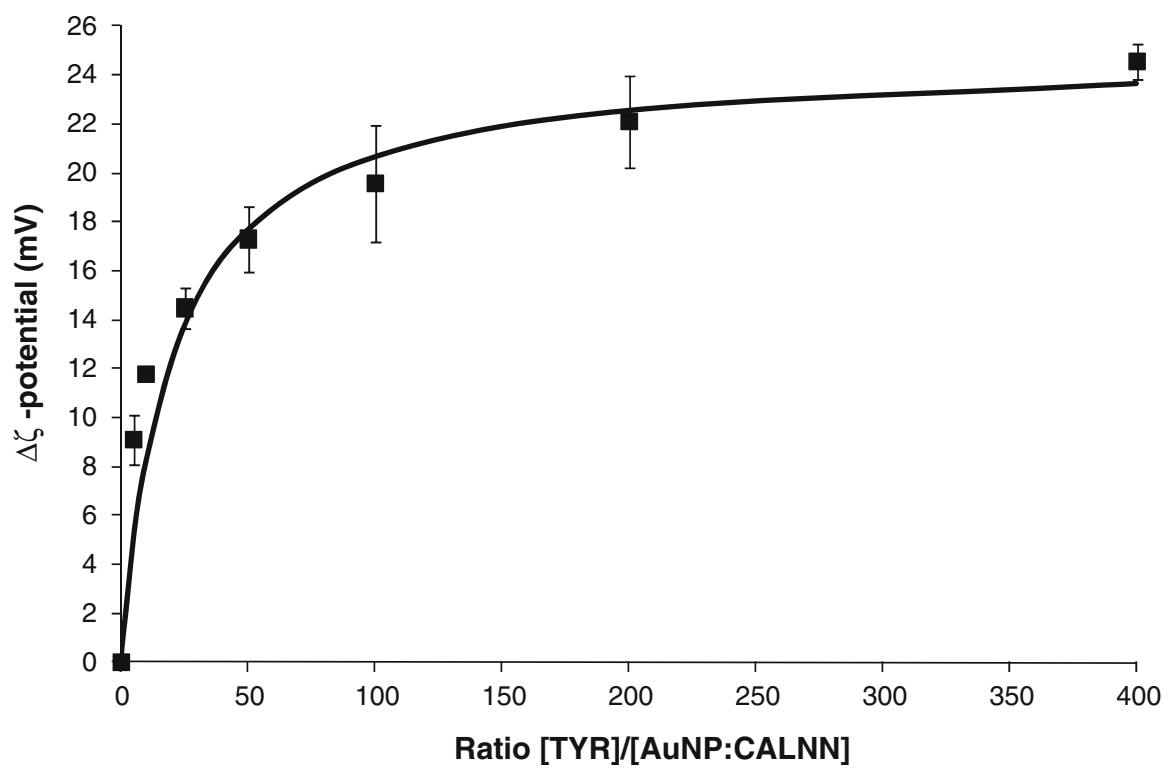


surface is saturated with protein, a similar approach as utilised for the $\zeta$-potential experiments.

An average hydrodynamic diameter of 10.4 $\pm 1.0 \mathrm{~nm}$ was observed for AuNP:CALNN alone. Increasing the ratios resulted in a shift of the average hydrodynamic diameter from the value of AuNP:CAL$\mathrm{NN}$ alone, to a maximal value $\max \mathrm{d}(H)=16.8$ $\pm 0.9 \mathrm{~nm}$ (as obtained from fitting to Eq. 3). Visual inspection of the graphical representation on Fig. 5 indicates that a maximal variation of $\mathrm{d}(H)$ is attained for a [TYR]/[AuNP:CALNN] ratio of approximately 50; and that no appreciable variation in diameter was observed for higher amounts of protein on the BNCs. This stabilisation of the hydrodynamic diameter values of the BNCs suggests that the AuNP surface becomes fully enveloped with a TYR monolayer, and no further absorption of protein occurs for ratios of [TYR]/ [AuNP:CALNN] above 50. This correlates well with the findings for $\zeta$-potential, supporting the formation of a protein monolayer surrounding the AuNP and thus of BNCs of TYR-AuNP:CALNN. The binding constant obtained from the fitting of Eq. 4 to the experimental data was $K_{\mathrm{L}}=0.052 \pm 0.006$, similar to that obtained for $\zeta$-potential.

$\Delta \mathrm{d}(H)=\frac{\Delta d(H) \max K_{\mathrm{L}} R}{K_{\mathrm{L}} R}$

in which $\Delta \mathrm{d}(H)$ is the variation in the average hydrodynamic diameter measured for a BNC with a surface coverage $R=[\mathrm{TYR}] /[\mathrm{AuNP}: \mathrm{CALNN}]$ in relation to the AuNP:CALNN alone; $\Delta \mathrm{d}(H) \max$ is the maximum value for the hydrodynamic diameter as $R$ increases; and $K_{\mathrm{L}}$ is a binding constant corresponding to the value of the inverse of the concentrations ratio, $1 / R$, for one-half of $\Delta \mathrm{d}(H) \max$.

Tyrosinase activity of the bionanoconjugates

The information obtained from $\zeta$-potential and average hydrodynamic diameter was used in the preparation of bionanoprobes, to ensure that the maximum amount of protein was loaded onto the AuNP and concurrently there was a minimum amount of free protein in solution (not bound to the nanoparticle, and thus not accountable as conjugate). The BNCs were thus prepared for a ratio [TYR]/[AuNP:CALNN] of $1: 100$, and their enzymatic activity was determined for $\mathrm{pH}$ range 4.0-8.0 and compared with that of the free enzyme (at the same concentration of active enzyme). This determination allowed obtaining the $\mathrm{pH}$ operational range of the BNCs in the sensing of phenolic compounds.

Analogous to the free enzyme, the BNCs presented a similar TYR activity pattern for $\mathrm{pH}$ 5.5-7.0, with a small reduction for $\mathrm{pH}$ 5.0. The enzyme activity was significantly lower for $\mathrm{pH} 4.5$ and 4.0, like the free TYR (Fig. 6). However, and more interestingly, when compared to the activity of the free enzyme, the BNCs
Fig. 5 Variation in the hydrodynamic diameters of the tyrosinase BNCs as a function of the [TYR]/ [AuNP:CALNN] ratio

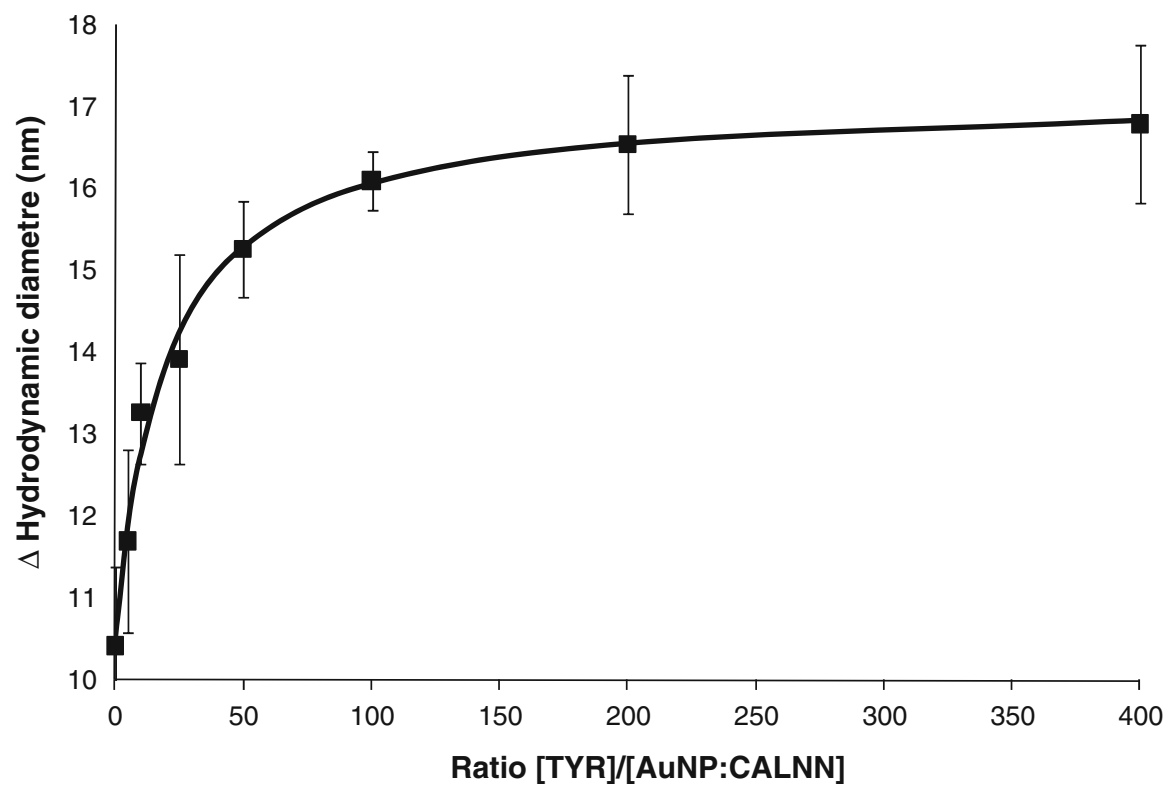


Fig. 6 Tyrosinase activities of the bionanoconjugates (BNCs) and of free enzyme (TYR), in the $\mathrm{pH}$ range $4-8$, using 4-methyl catechol as absorption maximum of the reaction product, $400 \mathrm{~nm}$ ). BNCs were prepared with the same enzyme units as those used for the experiments with free TYR substrate (expressed as absorption units at the



Fig. 7 Percentage increase in tyrosinase activity for the bionanoconjugates (BNCs) compared to the enzyme alone, for $\mathrm{pH}$ values in the 4-8 range. Blue bars are for solutions with aggregated BNCs; whereas red bars represent solutions in which aggregation does not occur. BNCs were prepared with the same enzyme units as those used for the experiments with TYR alone

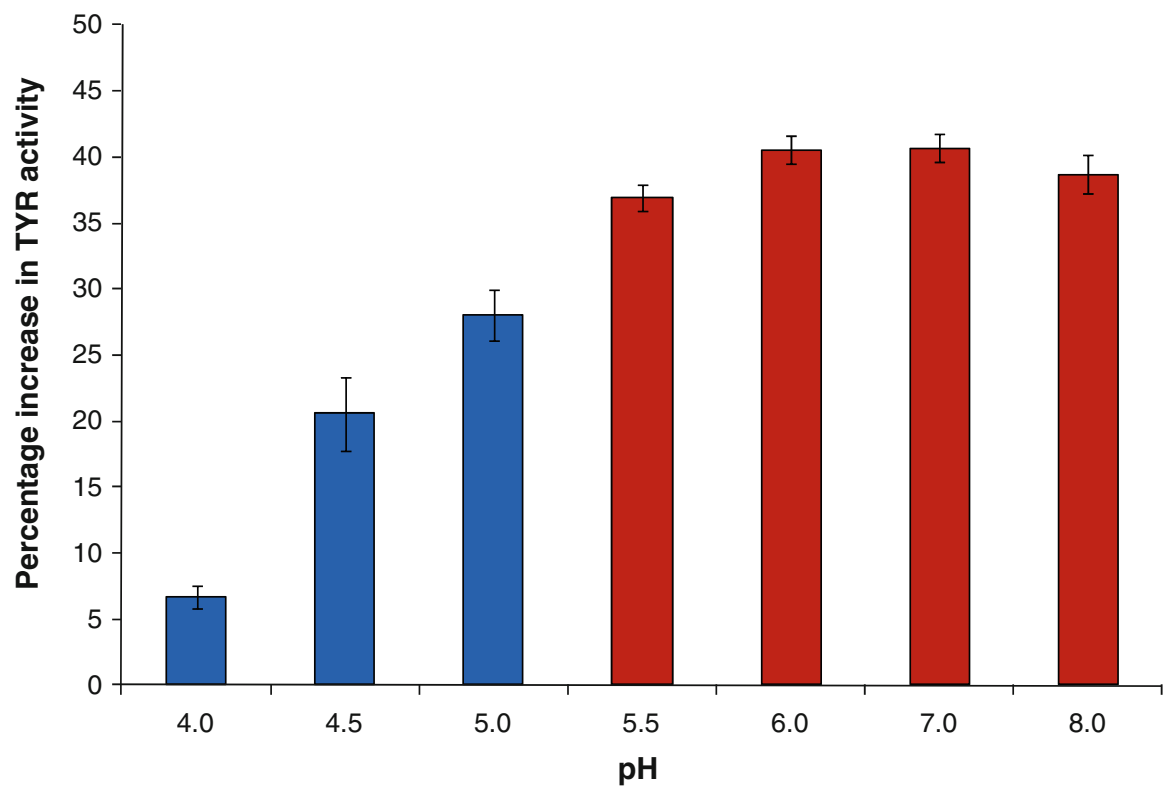

revealed activities $37-41 \%$ greater than those determined for the free enzyme for $\mathrm{pH}$ between 5.5 and 8.0 (Fig. 7). Furthermore, the activity of the BNCs at the optimum $\mathrm{pH}$ for the enzyme was $41 \%$ greater than that of the free enzyme. A possible explanation to the apparent increase in enzyme activity for the BNCs may be due to small alterations in the protein structure of TYR, leading to improved activity.

For $\mathrm{pH}$ range 5.0-4.0 this increase in activity was smaller. This indicates that the immobilisation of TYR on the surface of CALNN:AuNPs made the enzyme more sensitive to changes in $\mathrm{pH}$, but due to the increase in the activity of the enzyme in the $\mathrm{BNCs}$, the $\mathrm{pH}$ range where the nanobioconjugates show a useful activity was actually wider than for the native enzyme alone.

An increase of activity was also observed for TYR immobilised at the surface of citrate-capped AuNPs, but in this case the increase was lower $(20 \%$ at $\mathrm{pH}$ 7.0, in similar experimental conditions) showing that the pentapeptide provides a better local environment for enzyme adsorption. 
The determination of the activity of the BNCs for different $\mathrm{pH}$ values allowed the parallel assessment with the BNC aggregation phenomena (shown in Fig. 2). Interestingly, acidic $\mathrm{pH}$ values (below 5) correspond to smaller activity values both for TYR alone and for the BNCs; and also correspond to $\mathrm{pH}$ at which BNCs are aggregated (inset in Fig. 2). Nevertheless, and although the spectra for $\mathrm{pH} 4.5$ and 5.0 indicated aggregation, the BNCs retained TYR activity, in particular for $\mathrm{pH}$ 5.0. This is illustrated in Fig. 7, where the percentage increase in TYR activity of the BNCs was plotted with the aggregation curve of the BNCs for $\mathrm{pH} \mathrm{3-8.} \mathrm{This} \mathrm{indicated} \mathrm{that}$ BNC aggregation may not necessarily lead to a complete inhibition of TYR activity, suggesting that the active conformation of the enzyme when bound to the nanoparticle was conserved, and that most active sites remained accessible to the substrate.

The kinetic Michaelis-Menten constants $\left(K_{\mathrm{M}}\right.$ and $\left.V_{\max }\right)$ were determined for both the free enzyme and the BNCs at $\mathrm{pH} 7.0$, and were found to be similar (Table 1). The differences are not statistically significant $(p<0.05)$, indicating no obvious differences between the free and immobilised enzyme.

\section{Conclusions}

In summary, we examined the characteristics of CALNN-functionalised AuNPs and have demonstrated the formation of TYR-AuNP:CALNN BNCs, showing that the enzyme not only remains active after conjugation, but the enzyme activity in the BNCs is also higher than for the enzyme alone. The effective conjugation of proteins and AuNPs has been demonstrated for different proteins, including albumin (BSA) and Cytochrome c (Brewer et al. 2005; Gomes et al. 2008). The formation of BNCs was supported by the 3-nm red-shift to $525 \mathrm{~nm}$ observed in the plasmon resonance band of the BNCs relative to the functionalised AuNPs alone. Moreover, the decrease of the

Table 1 Michaelis-Menten kinetic parameters for the BNCs and the free enzyme for $\mathrm{pH} 7.0$

\begin{tabular}{lll}
\hline & $K_{\mathrm{M}}$ & $V \max$ \\
\hline Bionanoconjugates & $0.46 \pm 0.27$ & $1.44 \pm 0.67$ \\
Tyrosinase & $0.43 \pm 0.21$ & $1.52 \pm 0.54$ \\
\hline
\end{tabular}

surface charge of the BNCs as determined by zeta potential and the increase in hydrodynamic diameter determined by DLS further substantiate BNC formation. The difference in $\mathrm{pH}$-induced aggregation behaviour of the BNCs when compared to AuNP:CALNN alone also confirmed the formation of a stable protein corona around the AuNP:CALNN.

The conjugation with AuNPs generated BNCs with higher TYR activity than the enzyme alone at the same $\mathrm{pH}$, suggesting the AuNP:CALNN provide a suitable platform for the immobilisation of the enzyme, leading to more active nanoprobes with a wider $\mathrm{pH}$ working range for use in electrochemical biosensors for the detection of mono- and di-phenolic compounds. Moreover, the BNCs presented good storage stability, marginally higher than that of the free enzyme.

As described above, there are different approaches to producing AuNP-based biosensors. In particular, TYR biosensor conformations proposed thus far using AuNPs involve the separate deposition of AuNPs at an electrode with the subsequent immobilization of TYR. Kim et al. (2008a, c) proposed the electrodeposition of AuNPs followed by deposition of a SAM of thiolated ligand (containing either a carboxyl or an amine group at the extremity), and followed by the cross-linking of TYR via EDC-NHS. Immobilization via cross-linking is known to lead to loss of enzyme action, and thereby to biosensor sensitivity.

The approach proposed here of producing AuNP:CALNN-TYR BNCs has led to the stabilisation of the enzyme and to greater activity in solution when compared to the enzyme alone at the same concentration. Since conjugation is expected to involve mostly electrostatic interactions between the AuNP:CALNN and the enzyme protein, this seems to have resulted in the maintenance and even increase in enzyme activity compared to enzyme alone. A possible explanation to the apparent increase in enzyme activity in the BNCs may be due to small alterations in, for instance, the protein structure of the TYR, leading to improved activity.

The BNCs here produced can be deposited onto electrode surfaces, leading to greater enzyme activity in the deposits, and consequently to increased electrochemical signal generation and sensitivity compared to current conformations. Studies are underway to explore the possibility of depositing these BNCs on electrodes to produce highly active and sensitive TYR biosensors. 


\section{References}

Abdullah J, Ahmad M, Karuppiah N, Heng LY, Sidek H (2006) Immobilization of tyrosinase in chitosan film for an optical detection of phenol. Sens Actuators B Chem 114:604-609

Baptista P, Doria G, Henriques D, Pereira E, Franco R (2005) Colorimetric detection of eukaryotic gene expression with DNA-derivatized gold nanoparticles. J Biotechnol 119:111-117

Baptista P, Pereira E, Eaton P, Doria G, Miranda A, Gomes I, Quaresma P, Franco R (2008) Gold nanoparticles for the development of clinical diagnosis methods. Anal Bioanal Chem 391(3):943-950

Baron R, Willner B, Willner I (2007) Biomolecule-nanoparticle hybrids as functional units for nanobiotechnology. Chem Commun 28:323-332

Brewer SH, Glomm WR, Johnson MC, Knag MK, Franzen S (2005) Probing BSA binding to citrate-coated gold nanoparticles and surfaces. Langmuir 21:9303-9307

Dzyadevych SV, Soldatkin AP, Arkhypova VN, El'skaya AV, Chovelon JM, Georgiou CA, Martelet C, JaffrezicRenault N (2005) Early-warning electrochemical biosensor system for environmental monitoring based on enzyme inhibition. Sens Actuators B Chem 105:81-87

Fields GB, Noble RL (1990) Solid-phase peptide-synthesis utilizing 9-fluorenylmethoxycarbonyl amino-acids. Int J Pept Protein Res 35:161-214

Gomes I, Santos NC, Oliveira LMA, Quintas A, Eaton P, Pereira E, Franco R (2008) Probing surface properties of cytochrome $\mathrm{c}$ at $\mathrm{Au}$ bionanoconjugates. J Phys Chem $\mathrm{C}$ 112:16340-16347

Hedenmo M, Narvaez A, Dominguez E, Katakis I (1997) Improved mediated tyrosinase amperometric enzyme electrodes. J Electroanal Chem 425:1-11

Huang M, Ho T, Lee C (1992) Phenolic compounds in food and their effects on health. Antioxidants and cancer prevention. ACS, Washington

Jolivet S, Arpin N, Wichers HJ, Pellon G (1998) Agaricus bisporus browning: a review. Mycol Res 102:1459-1483

Kaiser E, Colescot RL, Bossinge CD, Cook PI (1970) Color test for detection of free terminal amino groups in solid-phase synthesis of peptides. Anal Biochem 34:595-598

Kaufman E et al (2007) Probing protein adsorption onto mercaptoundecanoic acid stabilized gold nanoparticles and surfaces by quartz crystal microbalance and zeta-potential measurements. Langmuir 23:6053-6062

Kim GY, Kang MS, Shim J, Moon SH (2008a) Substratebound tyrosinase electrode using gold nanoparticles anchored to pyrroloquinoline quinone for a pesticide biosensor. Sens Actuators B Chem 133:1-4

Kim GY, Shim J, Kang MS, Moon SH (2008b) Preparation of a highly sensitive enzyme electrode using gold nanoparticles for measurement of pesticides at the ppt level. J Environ Monitor 10:632-637

Kim GY, Shim J, Kang MS, Moon SH (2008c) Optimized coverage of gold nanoparticles at tyrosinase electrode for measurement of a pesticide in various water samples. J Hazard Mater 156:141-147
Kimling J, Maier M, Okenve B, Kotaidis V, Ballot H, Plech A (2006) Turkevich method for gold nanoparticle synthesis revisited. J Phys Chem B 110(32):15700-15707

Levy R (2006) Peptide-capped gold nanoparticles: towards artificial proteins. Chembiochem 7:1141-1145

Levy R, Thanh NTK, Doty RC, Hussain I, Nichols RJ, Schiffrin DJ, Brust M, Fernig DG (2004) Rational and combinatorial design of peptide capping Ligands for gold nanoparticles. J Am Chem Soc 126:10076-10084

Love JC, Estroff LA, Kriebel JK, Nuzzo RG, Whitesides GM (2005) Self-assembled monolayers of thiolates on metals as a form of nanotechnology. Chem Rev 105:1103-1169

Pingarron JM, Yanez-Sedeno P, Gonzalez-Cortes A (2008) Gold nanoparticle-based electrochemical biosensors. Electrochim Acta 53:5848-5866

Robb D (1984) Tyrosinase. In: Lontie R (ed) Copper proteins and copper enzymes, vol 2. CRC Press Inc., Boca Raton, pp 207-240

Rodriguez-Lopez J, Tudela J, Varon R, Garcia-Carmona F, Garcia-Canovas F (1992) Analysis of a kinetic model for melanin biosynthesis pathway. J Biol Chem 267: 3801-3810

Rogers KR (1995) Biosensors for environmental applications. Biosens Bioelectron 10:533-541

Salimi A, Sharifi E, Noorbakhsh A, Soltanian S (2007) Immobilization of glucose oxidase on electrodeposited nickel oxide nanoparticles: direct electron transfer and electrocatalytic activity. Biosens Bioelectron 22: 3146-3153

Selinheimo E, Gasparetti C, Mattinen M, Steffensen C, Buchert J, Kruus K (2009) Comparison of substrate specificity of tyrosinases from Trichoderma reesei and Agaricus bisporus. Enzyme Microb Technol 44:1-10

Sfinchez-Ferrer A, Rodriguez-Lopez J, Garcia-Cfinovas F, Garcia-Carmona F (1995) Tyrosinase: a comprehensive review of its mechanism. Biochim Biophys Acta 1247: $1-11$

Smith PK, Krohn RI, Hermanson GT, Mallia AK, Gartner FH, Provenzano MD, Fujimoto EK, Goeke NM, Olson BJ, Klenk DC (1985) Measurement of protein using bicinchoninic acid. Anal Biochem 150:76-85

Vamvakaki V, Chaniotakis NA (2007) Pesticide detection with a liposome-based nano-biosensor. Biosens Bioelectron 22:2848-2853

VanGelder CWG, Flurkey WH, Wichers HJ (1997) Sequence and structural features of plant and fungal tyrosinases. Phytochemistry 45:1309-1323

Vidal JC, Esteban S, Gil J, Castillo JR (2006) A comparative study of immobilization methods of a tyrosinase enzyme on electrodes and their application to the detection of dichlorvos organophosphorus insecticide. Talanta 68: 791-799

Viveros L, Paliwal S, McCrae D, Wild J, Simonian A (2006) A fluorescence-based biosensor for the detection of organophosphate pesticides and chemical warfare agents. Sens Actuators B Chem 115:150-157

Wang SS (1973) Para-Alkoxybenzyl alcohol resin and paraAlkoxybenzyloxycarbonylhydrazide resin for solid-phase synthesis of protected peptide fragments. J Am Chem Soc 95:1328-1333 
Willner I, Baron R, Willner B (2007) Integrated nanoparticlebiomolecule systems for biosensing and bioelectronics. Biosens Bioelectron 22:1841-1852

You CC, Verma A, Rotello VM (2006) Engineering the nanoparticle-biomacromolecule interface. Soft Matter 2:190-204
Yu HH, Liu SQ, Ju HX (2003) Mediator-free phenol sensor based on titania sol-gel encapsulation matrix for immobilization of tyrosinase by a vapor deposition method. Biosens Bioelectron 19:509-514 